\title{
Seismological Effects on Spectral and Pseudospectral Acceleration Proximities Based on Random Vibration Theory
}

Haizhong Zhang

Kanagawa University

Yan-gang Zhao ( $\nabla$ zhao@kanagawa-u.ac.jp)

Kanagawa University

\section{Research Article}

Keywords: Spectral absolute acceleration, pseudospectral acceleration, random vibration theory, seismological effects, inertial force.

Posted Date: July 20th, 2021

DOI: https://doi.org/10.21203/rs.3.rs-713123/v1

License: (c) (i) This work is licensed under a Creative Commons Attribution 4.0 International License.

Read Full License 


\section{Seismological effects on spectral and pseudospectral acceleration proximities based on random vibration theory}

\author{
Haizhong Zhang ${ }^{1)}$ and Yan-Gang Zhao ${ }^{1 *}$
}

\begin{abstract}
7 Although spectral absolute acceleration, $S a$, is a useful tool for estimating the inertial forces of
Abstract

structures in seismic designs, seismic codes typically specify only the pseudospectral acceleration, Spa. Many studies have been performed to clarify the relationship between these two spectra to relate them. A recent study indicated that this relationship could be affected by not only the structural damping ratio and period but also seismological parameters, such as magnitude and distance. However, how seismological parameters affect their relationship is not clearly understood. To clarify this issue, an approach that relates the two spectra, including seismological parameters, is proposed herein based on random vibration theory. The proposed approach is verified by comparison with the results of time-series analysis. Furthermore, the seismological effects are then explored and explained based on the proposed approach. It is found that $S p a$ approaches $S a$ with increasing moment magnitude and source-to-site distance, particularly at long structural periods, with the main reason being attributable to the increase in the long-period components of earthquake ground motions. Finally, a practical formulation for estimating $S a$ from $S p a$ considering the seismological effects is constructed and verified using real seismic records.
\end{abstract}

\section{Keywords}

Spectral absolute acceleration, pseudospectral acceleration, random vibration theory,

1) Department of Architecture, Kanagawa University, 3-27-1 Rokkakubashi, Kanagawa-ku, Yokohama, 221-8686, Japan

* Corresponding author: zhao@kanagawa-u.ac.jp 
seismological effects, inertial force.

\section{Introduction}

Response spectrum is presently the most widely used tool to characterize seismic loads for structural seismic designs. In most seismic codes, particularly those involving force-based design, the response spectrum for the design is typically specified as the 5\%-damped pseudospectral acceleration, $S p a$, along with a damping modification factor for adjustment to other damping levels (Pu et al., 2016; Zhang and Zhao, 2020). Because Spa is defined according to the spectral relative displacement, $S d$, as $S p a=\bar{\omega}^{2} S d$, with $\bar{\omega}$ being the structural circular frequency, the force estimated using Spa is proportional to the relative displacement, thus corresponding to the restoring force of the structure. Therefore, Spa is suitable in cases where the restoring force is of interest in the seismic design, e.g., in the design of structures where the damping is derived from the added energy dissipation devices (Lin and Chang, 2003). Nevertheless, when the inertial force is considered in the seismic design, e.g., to calculate the base shear in the designs of base-isolated structures or in foundation designs, the spectral absolute acceleration, $\mathrm{Sa}$, is more suitable (Sadek et al., 2000; Mentrasti, 2008).

Early studies have shown that for small structural damping ratios, $S a$ can be approximated by $S p a$; however, when the structural damping ratio is large and the structural period is long, $S a$ can significantly differ from Spa (Jenschke et al., 1964, 1965; Veletsos and Newmark, 1964; Newmark and Rosenblueth, 1971; Chopra, 2007) and is always greater than Spa (Newmark and Rosenblueth, 1971). Therefore, in such cases, directly using Spa to approximate $S a$ may underestimate the inertial forces and negatively affect the structural design.

47 Many studies have been conducted to clarify the relationship between $S a$ and Spa to relate them. Sadek et al. (2000) compared these two spectra based on statistical analyses of 72 accelerograms from 36 stations in western United States. Song et al. (2007) derived an 
analytical equation to relate the two spectra by assuming earthquake excitation as a Gaussian stationary process. Mentrasi (2008) theoretically analyzed and explained the relationship between these two spectra based on exact integral analysis. Zhang et al. (2016) discussed these spectra and compared them based on the El Centro earthquake record.

The above studies have yielded useful results on the relationship between the two spectra as well as the effects of the structural parameters, namely structural period and damping ratio. Papagiannopoulos et al. (2013) further noted that the relationship between them is affected by not only the structural but also seismological parameters, e.g., magnitude and distance, based on the statistical analyses of 866 accelerograms recorded worldwide. However, the principle underlying how the seismological parameters affect the relationship between the two spectra has not been elucidated.

The aim of this paper is to clarify such seismological effects. The remainder of this paper is organized as follows. First, an approach to relate $S a$ and Spa including seismological parameters is developed based on random vibration theory (RVT). This approach is then verified by comparison with the results of time-series analysis. Subsequently, based on the developed approach, the seismological effects on the relationship between the two spectra are systematically investigated and theoretically explained. Further, a practical formulation for estimating $S a$ from $S p a$ considering the seismological effects is constructed and verified using real seismic records. Finally, the conclusions of this study are shown.

\section{Approach to relate $S a$ and $S p a$}

To investigate the seismological effects on the relationship between $S a$ and $S p a$, there are two options: using real accelerograms or ground-motion prediction models. To identify consistent patterns within an appreciable margin of variability that is always displayed by strong-motion data, large numbers of records must be employed because one is unlikely to find 
records that are essentially similar in all but one characteristic. Therefore, the ground-motion prediction models for $S p a$ and $S a$ are more suitable for use in this study. Although there are many available ground-motion prediction models for the 5\%-damped Spa, very few are available for 5\%-damped $S a$, and even fewer for $S a$ with various damping ratios. In this study, we adopt a Fourier amplitude spectrum (FAS) ground-motion model and RVT to estimate and relate the two spectra considering the ability of the RVT to relate the FAS to the response spectrum at any desired damping level. The proposed analytical approach allows the investigation of the seismological effects as well as a theoretical explanation for the observed phenomena.

\subsection{Earthquake ground-motion model}

Various methods are available in the literature to describe the FAS of the earthquake ground motions. The simplest method involves using seismology theory to compute the radiated FAS from a point source in terms of the various source, path, and site parameters. This study utilizes the seismological point-source theory to derive the FAS based on the description by Boore (2003). The FAS of the ground-motion acceleration, $Y(f)(\mathrm{cm} / \mathrm{s})$, can be expressed as

$$
Y(f)=\left[0.78 \frac{\pi}{\rho \beta^{3}} M_{0} \frac{f^{2}}{1+\left(f / f_{c}\right)^{2}}\right]\left[Z(R) \times \exp \left(\frac{-\pi f R}{Q(f) \beta}\right)\right]\left[\exp \left(-\pi \kappa_{0} f\right) A(f)\right]
$$

where $f=$ frequency $(\mathrm{Hz}) ; \rho=$ mass density of the crust $\left(\mathrm{g} / \mathrm{cm}^{3}\right) ; \beta=$ shear-wave velocity of the crust $(\mathrm{km} / \mathrm{s}) ; R=$ distance from the source $(\mathrm{km}) ; Z(R)=$ geometric attenuation; $\kappa_{0}=$ site diminution (s); $Q(f)=$ anelastic attenuation; $A(f)=$ crust amplification; $M_{0}=$ seismic moment (dyne $\mathrm{cm}$ ), which is related to the moment magnitude, $M$, as $M_{0}=10^{1.5 M+10.7} ; f_{c}=$ corner frequency given as $f_{c}=4.9 \times 10^{6} \beta\left(\Delta \sigma / M_{0}\right)^{1 / 3}$, which represents the frequency below which the FAS decays; $\Delta \sigma=$ stress drop. The seismological parameters required in Eq. (1) for central and eastern North America (CENA) are used in this study because of recent work that have updated 
the seismological parameters for these regions (Boore and Thompson, 2015), as detailed in Table 1 of Wang and Rathje (2016).

\subsection{Expression for $\mathrm{Sa} / \mathrm{Spa}$}

Based on the RVT, both $S a$ and $S p a$ can be obtained from the FAS at any desired damping level. The RVT states that the peak value of a time-series signal is equal to the product of the peak factor and root-mean-square (rms) value, which can be expressed as

$$
a_{\max }=p f \sqrt{\frac{1}{D \pi} \int_{0}^{\infty}|y(\omega)|^{2} d \omega}
$$

where $a_{\max }$ is the peak value of the signal, $p f$ denotes the peak factor, and the square-root part in Eq. (2) represents the rms value of the signal, which is obtained from the signal duration $D$ and FAS of the signal $y(\omega)$, and $\omega$ is the circular frequency $(\omega=2 \pi f)$. Since the response spectrum is the peak response value of a single-degree-of-freedom (SDOF) oscillator, according to RVT, the response spectrum should be equal to the product of the peak factor and rms of the oscillator response. Boore (2003) derived an expression for Spa, which is expressed as

$$
\operatorname{Spa}(\bar{\omega}, \xi)=p f_{\bar{\xi} p} \sqrt{\frac{1}{D_{\mathrm{rms}} \pi} \int_{0}^{\infty}|Y R(\omega, \bar{\omega}, \xi)|^{2} d \omega}
$$

where, $\bar{\omega}$ and $\xi$ are the SDOF-oscillator circular frequency and damping ratio, respectively; $p f_{\tilde{s}}$ is the peak factor of the oscillator response, and the square-root part in Eq. (3) represents the rms value of the oscillator response, which is obtained using the oscillator-response duration, $D_{\mathrm{rms}}$, and oscillator-response FAS, $Y R(\omega, \bar{\omega}, \xi)$. Here, $Y R(\omega, \bar{\omega}, \xi)$ is equal to the product of the ground-motion FAS $Y(\omega)$ and modulus of the oscillator transfer function for Spa, $H p a(\omega, \bar{\omega}, \xi)$, i.e., $\quad \operatorname{YR}(\omega, \bar{\omega}, \xi)=Y(\omega)|H p a(\omega, \bar{\omega}, \xi)| . \quad H p a(\omega, \bar{\omega}, \xi)$ is expressed as

$$
|H p a(\omega, \bar{\omega}, \xi)|=\frac{1}{\sqrt{(2 \xi \omega / \bar{\omega})^{2}+\left((\omega / \bar{\omega})^{2}-1\right)^{2}}}
$$


117 The oscillator transfer function for $\operatorname{Spa}, \operatorname{Hpa}(\omega, \bar{\omega}, \xi)$, is obtained from the one for $S d$, $118 H d(\omega, \bar{\omega}, \xi)$, by multiplication with $\bar{\omega}^{2}$, i.e., $|H p a(\omega, \bar{\omega}, \xi)|=\bar{\omega}^{2}|H d(\omega, \bar{\omega}, \xi)| . H d(\omega, \bar{\omega}, \xi)$

119 is expressed as

$$
|H d(\omega, \bar{\omega}, \xi)|=\frac{1 / \bar{\omega}^{2}}{\sqrt{(2 \xi \omega / \bar{\omega})^{2}+\left((\omega / \bar{\omega})^{2}-1\right)^{2}}}
$$

It should be noted that in RVT analysis, multiplying the spectral relative displacement $S d$ by $\bar{\omega}^{2}$ to obtain Spa is equivalent to multiplying the oscillator transfer function $H d(\omega, \bar{\omega}, \xi)$ for $S d$ by $\bar{\omega}^{2}$ to obtain Spa. This is attributed to the property of Eq. (3), in which the integral of $Y R(\omega, \bar{\omega}, \xi)$ is related to $\omega$; thus, one can insert $\bar{\omega}^{2}$ into the integral, i.e., transform $H d(\omega, \bar{\omega}, \xi)$, or outside the radical sign, i.e., transform $S d$, to obtain the same results.

Similarly, based on the RVT, $S a$ can be obtained by replacing the oscillator transfer function for $S p a$ in Eq. (3) by that for $S a$. The oscillator transfer function for $S a, H a(\omega, \bar{\omega}, \xi)$, is given 127 by

$$
|H a(\omega, \bar{\omega}, \xi)|=\frac{\sqrt{(2 \xi \omega / \bar{\omega})^{2}+1}}{\sqrt{(2 \xi \omega / \bar{\omega})^{2}+\left((\omega / \bar{\omega})^{2}-1\right)^{2}}}
$$

Then, the expression for the ratio of $S a$ to $S p a$ can be obtained using the respective spectra:

$$
\frac{\operatorname{Sa}(\bar{\omega}, \xi)}{\operatorname{Spa}(\bar{\omega}, \xi)}=\sqrt{\frac{\int_{0}^{\infty}|Y(\omega) H a(\omega, \bar{\omega}, \xi)|^{2} d \omega}{\int_{0}^{\infty}|Y(\omega) H p a(\omega, \bar{\omega}, \xi)|^{2} d \omega}} \times \frac{p f_{\xi}}{p f_{\tilde{\xi} p}}
$$

where $p f_{\xi}$ is the oscillator-response peak factor corresponding to $S a$. Thus, Eq. (7) theoretically relates to the two spectra. Here, it should be noted that the oscillator-response duration for $S a$ is assumed to be the same as that for $S p a$ in the derivation of Eq. (7). The expression for $S a / S p a$ in Eq. (7) can be decomposed into two terms: the first term (i.e., $\sqrt{\int_{0}^{\infty}|Y(\omega) H a(\omega, \bar{\omega}, \xi)|^{2} d \omega /}$ 
hereafter denoted as $R_{\mathrm{rms}}$; and the second term (i.e., $p f_{\xi} p f_{\xi p}$ ) is the ratio of the oscillator-response peak factors for $S a$ and $S p a$, hereafter denoted as $R_{p f}$.

When applying Eq. (7) to estimate the ratio of the two spectra, the value of the peak factor must be determined. Many models have been developed for the estimation of the peak factor (Cartwright and Longuet-Hlggms, 1956; Davenport, 1964; Vanmarcke, 1975). Among these, the model by Vanmarcke (1975) has been found to provide the most reasonable estimations of the response spectra in RVT analysis (Wang and Rathje, 2016). The cumulative distribution function, $P$, of the peak factor, $p f$, as provided by Vanmarcke (1975) is expressed as

$$
P(p f<r)=\left[1-e^{\left(-r^{2} / 2\right)}\right] \times \exp \left[-2 f_{z} \exp \left(-r^{2} / 2\right) D_{g m} \frac{\left(1-e^{-\delta^{1.2} r \sqrt{\pi / 2}}\right)}{\left(1-e^{r^{2} / 2}\right)}\right]
$$

Here, $\delta$ is a bandwidth factor, which is defined as a function of the spectral moments:

$$
\delta=\sqrt{1-\frac{m_{1}^{2}}{m_{0} m_{2}}}
$$

where $m_{0}, m_{1}$, and $m_{2}$ denote the zeroth-, first-, and second-order moments of the square of the FAS, and the nth-order spectral moment, $m_{n}$, is defined as

$$
m_{n}=\frac{1}{\pi} \int_{0}^{\infty} \omega^{n}|Y(\omega)|^{2} d \omega
$$

In addition, $f_{z}$ denotes the rate of zero crossings, which is also a function of the spectral moments, and is given by

$$
f_{z}=\frac{1}{2 \pi} \sqrt{\frac{m_{2}}{m_{0}}}
$$

The ground-motion duration $D_{g m}$ in Eq. (8) is estimated based on the model of Boore and Thompson $(2014,2015)$. In RVT analysis, the expect value of the peak factor is always used, which can be obtained using Eq. (8) by the expression $\int_{0}^{\infty}[1-P(p f<r)] d r$.

\section{Verification}


To investigate the accuracy of the proposed approach, the ratio of the two spectra, Sa/Spa, was calculated using Eq. (7) and compared with that obtained using traditional time-series analysis. The time series for the analysis was generated from ground-motion FAS using the stochastic method SIMulation (Boore, 2005) program via stochastic simulations (Boore, 1983). For each FAS, a suite of 100 time-series signals was generated, and the simulated time series matched the FAS on average. Then, the values of $S a, S p a$, and the spectral ratios $S a / S p a$ for all the generated time series were calculated using the direct integration method by Nigam and Jennings (1969). For each FAS, the 100 corresponding results of the spectral ratios Sa/Spa for a given damping level were averaged and compared with those obtained using the proposed approach. A wide range of the structural parameters, i.e., oscillator period $T_{0}(0.01-10 \mathrm{~s})$ and damping ratio $\xi(10-50 \%)$, as well as main seismological parameters, including the moment magnitude $M(4-8)$ and site-to-source distance $R(20-200.01 \mathrm{~km})$, were considered in the calculations. Some of these representative comparisons are shown in Figs. 1-3. The favorable agreement in these figures validate the proposed approach.

From the figures, the relationship between the two spectra can also be clearly observed. It is observed that the values of $S a / S p a$ are always greater than unity for all cases, which implies that $S a$ is always greater than $S p a$. Figure 1 also indicates that $S a / S p a$ is nearly equal to unity at small oscillator periods and increases with increasing oscillator periods and damping ratios; these values may be considerably greater than unity for very long oscillator periods and very large damping ratios. These observed properties for the relationship between the two spectra are consistent with those observed by statistical analyses of real seismic records and common knowledge (Jenschke et al., 1964, 1965; Veletsos and Newmark, 1964; Newmark and Rosenblueth, 1971; Sadek et al., 2000; Chopra, 2007; Song et al., 2007; Mentrasti, 2008; Papagiannopoulos et al., 2013; Zhang et al., 2016). The consistency of these observations provides additional confirmation of the proposed approach. 


\section{Seismological effects on $S a-S p a$ relationship}

\subsection{Exploration of the seismological effects}

This section investigates the seismological effects on the relationship between $S a$ and $S p a$ based on the proposed approach. From Figs. 2 and 3 in the previous section, the $S a / S p a$ trends for variation of the moment magnitude and site-to-source distance can be easily clarified. Figure 2 indicates that $S a / S p a$ is considerably affected by the moment magnitude. The value of Sa/Spa decreases with increase in the moment magnitude, particularly for long oscillator periods. Figure 3 indicates that the behavior of $S a / S p a$ with variation of the site-to-source distance $R$ is typically consistent with the variation of the moment magnitude but to a much smaller degree of variation.

The effects of the site conditions on the relationship between the two spectra were also investigated. The site term in the FAS model of Eq. (1) corresponds to crustal amplification for hard rock. To investigate the effects of the surface site conditions, three soft soil sites composed of single layers of soil on hard rock were considered. Further, two levels of the soil damping ratio, $h$ (1\% and 10\%), were considered. Values of Sa/Spa for the three soil sites were calculated using the proposed approach by considering all structural and seismological parameters in the previous section. The representative results corresponding to an oscillator damping ratio of $30 \%$ are shown in Fig. 4; additionally, the undamped site fundamental period, $T_{\mathrm{s}}$, impedance ratio of the soil layer with respect to hard rock, Ip, and soil damping ratio, $h$, are presented. It is evident from the figure that $S a / S p a$ varies with the soil parameters, i.e., impedance ratio, soil damping ratio, and site fundamental period, but to a much smaller degree than with the moment magnitude (Fig. 2). In addition, the effects of the site conditions on 
parameters of the soil sites. It is noted that Sa/Spa for the soil site in Fig. 4(b) is nearly the same as that for the rock site. When Ip is larger (Fig. 4(a)) or the site fundamental period $T_{\mathrm{S}}$ is longer (Fig. 4(c)), Sa/Spa for the soil site decreases compared with that for the rock site. When the soil damping ratio $h$ is smaller (Fig. 4(d)), Sa/Spa for the soil site increases compared with that for the rock site. In addition, from comparison of the results in Fig. 4, it is observed that the effect of the site fundamental period on Sa/Spa is the most significant compared with other site parameters.

\subsection{Explanation of the observed phenomenon}

The observed phenomena can be theoretically explained based on the proposed approach.

212 The representative results of the two terms in Eq. (7), i.e., $R_{\mathrm{rms}}$ and $R_{p f}$, for the cases in Fig. 2 are shown in Figs. 5 and 6 to investigate their contributions toward Sa/Spa. It is evident from Figs.

2 and 5 that the value of $S a / S p a$ is similar to that of $R_{\mathrm{rms}}$, and all the characteristics, including the trends with variations in the structural and seismological parameters, are captured adequately by $R_{\text {rms. }}$ In addition, Fig. 6 indicates that the value of $R_{p f}$ is close to unity compared to $R_{\text {rms. }}$. These observations indicate that $S a / S p a$ is dominated by $R_{\mathrm{rms}}$, which facilitates explanation of the phenomenon based on $R_{\mathrm{rms}}$.

To facilitate further analysis, the integral terms in the numerator and denominator of $R_{\mathrm{rms}}$, i.e.,

$\int_{0}^{\infty}|Y(\omega) H a(\omega, \bar{\omega}, \xi)|^{2} d \omega$ and $\int_{0}^{\infty}|Y(\omega) H p a(\omega, \bar{\omega}, \xi)|^{2} d \omega$, are regarded as areas of the square of the oscillator-response FAS enclosed by the circular frequency axis. The integral term in the numerator, i.e., $\int_{0}^{\infty}|Y(\omega) H a(\omega, \bar{\omega}, \xi)|^{2} d \omega$, corresponds to $S a$ and that in the denominator, i.e.,

$\int_{0}^{\infty}|Y(\omega) H p a(\omega, \bar{\omega}, \xi)|^{2} d \omega$, corresponds to Spa. Thus, the proximity between the two spectra or the value of $S a / S p a$ is determined by the proximity between these two areas. The closer the two areas are, the more similar are the two spectra, and the closer is the value of $S a / S p a$ to unity. It 
can be further observed from the first term $R_{\mathrm{rms}}$ in Eq. (7) that the difference between the two

227

areas is caused by the difference between the oscillator transfer functions of the two spectra, i.e., $H a(\omega, \bar{\omega}, \xi)$ and $\operatorname{Hpa}(\omega, \bar{\omega}, \xi)$, because the ground-motion FAS $Y(\omega)$, which affects the oscillator-response FAS, is the same for the two spectra.

To investigate the differences between the oscillator transfer functions for $S a$ and $S p a$, their values for two oscillator damping ratios, namely $5 \%$ and $30 \%$, are compared in Fig. 7 . The oscillator period was chosen as $0.5 \mathrm{~s}$ for comparison. Here, it should be noted that the oscillator period $T_{0}$ is different from the period $T(T=2 \pi / \omega)$ of the ground-motion FAS. It can be found that the two oscillator transfer functions are very similar for the smaller oscillator damping ratio $(5 \%)$. However, with the increase in the oscillator damping ratio, the difference between the two oscillator transfer functions increases over short periods. Nevertheless, at periods around and greater than the oscillator period $T_{0}$, the values of the two oscillator transfer functions are very similar. In addition, it should be noted that values of $\operatorname{Ha}(\omega, \bar{\omega}, \xi)$ are always greater than those of $\operatorname{Hpa}(\omega, \bar{\omega}, \xi)$, and the degree increases with increasing oscillator damping ratios, particularly for short periods. Based on these properties of the oscillator transfer functions, the $S a / S p a$ trends with variations in the oscillator damping ratio can be clarified. Because the difference between the two oscillator transfer functions increases with increasing oscillator damping ratio, the difference between the two integral areas, i.e., $\int_{0}^{\infty}|Y(\omega) H a(\omega, \bar{\omega}, \xi)|^{2} d \omega$ and $\int_{0}^{\infty}|Y(\omega) H p a(\omega, \bar{\omega}, \xi)|^{2} d \omega$, will increase. This causes an increase in the difference between the two spectra with increases in the oscillator damping ratios (Fig. 1). Further, because the value of the oscillator transfer function for $S a$ is always greater than that for Spa, $\int_{0}^{\infty}|Y(\omega) H a(\omega, \bar{\omega}, \xi)|^{2} d \omega>\int_{0}^{\infty}|Y(\omega) H p a(\omega, \bar{\omega}, \xi)|^{2} d \omega$, and the value of $S a$ is always larger than that of Spa (Fig. 1-3).

To explain the Sa/Spa trend with variation in oscillator period, the values of the two 
oscillator transfer functions for different oscillator periods ( 0.1 and $1 \mathrm{~s})$ are compared in Fig. 8.

251 The oscillator damping ratio is set as $30 \%$ for comparison. It is observed that owing to the increase in the oscillator period, the region of the oscillator transfer functions with periods less than the oscillator period $T_{0}$ increases. Because the values of these two transfer functions are very different in this region $(\operatorname{Ha}(\omega, \bar{\omega}, \xi)>\operatorname{Hpa}(\omega, \bar{\omega}, \xi))$, as shown in Fig. 8, the differences between the two areas given by the numerator $\int_{0}^{\infty}|Y(\omega) H a(\omega, \bar{\omega}, \xi)|^{2} d \omega$ and denominator $\int_{0}^{\infty}|Y(\omega) H p a(\omega, \bar{\omega}, \xi)|^{2} d \omega$ of $R_{\mathrm{rms}}$ increase with increasing oscillator periods. This explains the increase in the differences between the two spectra or the values of Sa/Spa with increasing oscillator periods (Figs. 1-3).

To explain the $S a / S p a$ trend with variations in the seismological parameters, namely the moment magnitude, site-to-source distance, and site conditions, the key factor governing the seismological effects is investigated in accordance with $R_{\mathrm{rms}}$ in Eq. (7). It is observed that the seismological parameters affect $R_{\mathrm{rms}}$ by changing the ground-motion FAS. The other components affecting the oscillator-response FAS in $R_{\text {rms }}$, i.e., the oscillator transfer functions, are independent of the seismological parameters. Moreover, it is known that the distribution of the ground-motion FAS with frequency instead of absolute values of the FAS affects the first term. This conclusion can be easily verified by multiplying the FAS by a constant value; since a constant value exists in both the numerator and denominator of $R_{\mathrm{rms}}$, they will cancel each other and will not affect $R_{\text {rms }}$. Therefore, the key factor affecting the seismological effects is the distribution of ground-motion FAS with frequency, i.e., the frequency content of the ground motions.

To investigate the variation of the frequency content of ground motions with the moment magnitude and site-to-source distance, the values of the ground-motion FAS for two moment magnitudes and two site-to-source distances are compared in Fig. 9. It is noted that although 
the FAS values at all periods increase with increasing moment magnitudes, the increases in the long periods are more significant. This indicates that the long-period components of the earthquake ground motions increase relatively compared to the short-period components with increasing moment magnitudes. Similarly, although the FAS values at all periods decrease with increasing site-to-source distances, the decreases at short periods are more significant. This means that the long-period components of the earthquake ground motions increase relative to the short-period components with increasing site-to-source distances. In addition, it is observed that the degree of variation of the frequency content of the ground motions with the site-to-source distances is smaller than that of the moment magnitude.

Based on the above properties of variation of the ground-motion frequency content with moment magnitude and site-to-source distance, the $S a / S p a$ variation trends can be explained. It is worth emphasizing again that the spectral ratio $S a / S p a$ can be understood by investigating the differences between the two areas of the square of the oscillator-response FAS in Eq. (7).

The difference between these two areas is attributed to the difference between their oscillator transfer functions over short periods. In addition, the two transfer functions are very similar at periods around and longer than the oscillator period $T_{0}$. It can be seen from Fig. 9 that when the components are concentrated more over a long period, in which the two oscillator transfer functions are similar, the two spectra will be more similar. Therefore, when the long-period components of the ground motions increase with increasing moment magnitudes and site-to-source distances, similar portions of the two areas will increase relatively, as shown in Fig. 9. This explains why Spa approaches $S a$ with increasing moment magnitudes and site-to-source distances (Fig. 2).

To explain the effects of site conditions on the relationship between the two spectra, the FAS

297 values for the soil sites in Fig. 4 are compared with those for the rock sites, which is shown in 
content are not as simple as those of the moment magnitude and site-to-source distance. The

300

301

302

303

304

305

306

307

308

309

310

311

312

313

314

315

316

317

318

319 site effect on the frequency content is strongly dependent on the period $T$. The components around the first few site resonance periods are amplified; however, those between these site resonance periods or at very short periods are de-amplified, as reported in previous studies (Zhang and Zhao, 2017, 2018). The components longer than the site fundamental period remain nearly unchanged. In addition, the amplification/de-amplification degree is dependent on the impedance ratio (Figs. 10 (a) and (b)) and soil damping ratio (Figs. 10 (c) and (d)). Moreover, the regions where the FAS values are de-amplified by the soil site in the shorter periods are strongly affected by the fundamental period of the site; as the site fundamental period increases, the de-amplification region becomes wider (Figs. 10 (b) and (c)).

Based on the properties of the effects of the site conditions on the frequency content, the Sa/Spa trends with variation of site conditions can be explained. When the impedance ratio and soil damping are large (Fig. 10 (a)) or the site fundamental period is long (Fig. 10 (c)), the decreases in the components in the short period are more significant than the increases in the components around the site resonance periods. This means that the short-period components of the ground motion decrease. Because the two oscillator transfer functions are different at short periods and are very similar at periods around and greater than the oscillator period $T_{0}$, when the short-period components of the ground motion decrease, the different parts of these two areas will decrease. Therefore, the difference between the two areas in $R_{\text {rms }}$ decreases, and Sa/Spa approaches unity, as shown in Figs. 4 (a) and (c). When the soil damping is very small (Fig. 10 (d)), the degree of increase in the components around the site resonance periods is more significant than the degree of decrease of the components over short periods. This implies that the short-period components of the ground motions increase. Therefore, the difference between the two areas in the first term increases, and Sa/Spa increases compared to that for the hard rock site, as shown in Fig. 4 (d). 


\section{Practical Sa/Spa formulation}

\subsection{Formulation construction}

Based on the conclusions derived in Section 4, this section constructs a simple Sa/Spa formulation considering the seismological effects for practical use in seismic design. To this end, a large number of function forms are trialed to fit the Sa/Spa results in Section 3. Finally, considering a balance between accuracy and simplicity, a simple Sa/Spa formulation is proposed, which is expressed as

$$
\frac{S a}{S p a}=1+\left(0.14 \xi^{1.54} \zeta^{-0.57}\right) T_{0}^{\xi^{-0.2 /(5 \sqrt{\zeta}+1)}}
$$

332

where $\zeta$ is a parameter reflecting the seismological effects on $S a / S p a$, which is detailed below. This equation satisfies the boundary condition that, when the oscillator period $T_{0}$ and damping ratio $\xi$ decrease to zero, Sa/Spa equals unity. Equation (12) also captures the $S a / S p a$ trends with variations in the oscillator period and damping ratio observed in Figs. 1-3 and previous studies using real seismic records (Sadek et al., 2000; Papagiannopoulos et al., 2013).

Because the seismological parameters (especially the moment magnitude $M$ ) can significantly influence $S a / S p a$, as observed above, Eq. (12) incorporates the seismological effects. In principle, the seismological parameters, e.g., the moment magnitude $M$ and site-to-source distance $R$, should be explicitly included in the Sa/Spa formulation. However, most of these parameters are not available in seismic codes, a formulation including such parameters is unrealistic for practical seismic design. Section 4 indicates that the seismological effects on Sa/Spa are governed by the frequency content of the ground motion, therefore, a parameter related to the frequency content can be adopted to reflect the seismological effects. Various frequency-content parameters have been defined based on the FAS and response spectra of ground motions (Craifaleanu, 2011). Because only Spa is 
typically specified in seismic codes, four parameters available from Spa, introduced by 349 Craifaleanu (2011) — specifically, the predominant period $T_{g S V}$, modified spectral

350

characteristic period $T^{*}{ }_{\text {mean }}$, modified central period $T^{*}{ }_{c e n}$, and modified shape factor $q^{*}-$ are tested. It is found that (not shown here), although the four parameters are closely related to moment magnitude and site-to-source distance, when they are applied to derive the $\mathrm{Sa} / \mathrm{Spa}$ formulation, the estimated results are not sufficiently stable. Therefore, a new frequency-content parameter $\zeta$ is proposed based on Spa to reflect the seismological effects. This parameter is expressed as

$$
\zeta=\frac{\operatorname{Spa}(6 \mathrm{~s})}{\mathrm{PGA}}
$$

where, Spa (6s) and PGA are the values of Spa at $6 \mathrm{~s}$ and peak ground acceleration (i.e., Spa at $0 \mathrm{~s}$ ) for a $5 \%$ damping ratio, respectively. It can be seen from Eq. (13) that $\zeta$ can be easily obtained from the Spa specified in seismic codes.

It can be easily known from Eq. (13) that $\zeta$ is closely related to the frequency content of the ground motion. When the short-period components are predominant and the bandwidth is narrow, $\operatorname{Spa}(6)$ is small relative to PGA, thus $\zeta$ is small. Conversely, when the bandwidth increases with the long-period component, $\operatorname{Spa}(6)$ increases relative to PGA, hence $\zeta$ will increase. Therefore, $\zeta$ typically increases with the long-period components and can simply reflect the frequency content of the ground motion. In fact, the use of the Spa values at periods 0 and $6 \mathrm{~s}$ was determined by trialing numerous values at different periods; it is found that these two values result in Eq. (12) generating the best precision.

The Sa/Spa results calculated by Eq. (12) were compared with those in Section 3; some representative comparisons are shown in Fig. 11. It is noted that Eq. (12) performs very well in the prediction of $S a / S p a$, particularly in the period range, $T_{0}<6 \mathrm{~s}$, which is generally of interest in practical seismic design. 


\subsection{Comparison with real seismic records}

The $S a / S p a$ results produced by Eq. (12) were further compared with those of real seismic records. As the seismological parameters in Eq. (1), e.g., stress drop and geometric attenuation, for CENA were used for the above analyses, for consistency, the seismic records for the verification were also selected from the regions of CENA. All seismic ground motions in the Pacific Earthquake Engineering Research Center (PEER) database from CENA were searched. A total of 367 seismic records (734 accelerograms) with moment magnitude larger than 4.0 and rupture distance between 10 to $300 \mathrm{~km}$ were selected. Because in the regions of CENA, there is a lack of large earthquakes, the largest moment magnitude of the selected records was limited to 5.85. The selected records were then classified into four groups considering a balance in the number of records, as shown in Fig. 12. It is evident that the Sa/Spa results by the proposed formulation are highly congruent with those of the real seismic records.

In addition, the Sa/Spa results obtained in previous studies (Sadek et al., 2000; Song et al. 2007; Mentrasi 2008) are plotted in Fig. 12. It is clear from the figure that the proposed formulation performs better than existing ones that do not incorporate the seismological effects. The $S a / S p a$ results produced by the existing formulation basically correspond to those with large moment magnitude.

\section{Conclusions}

To investigate the effects of seismological parameters on the relationship between spectral and pseudospectral accelerations, i.e., $S a$ and $S p a$, an approach relating the two spectra including seismological parameters is proposed based on random vibration theory. Using the proposed approach, the effects of the seismological parameters on the relationship between the two spectra are systematically explored and theoretically explained. Finally, a practical 
formulation for estimating $S a$ from $S p a$ considering the seismological effects is constructed. The main conclusions of this study are summarized as follows:

1. The comparisons with the results obtained from traditional time-series analyses show that the proposed approach is valid.

2. The relationship between the spectral absolute acceleration $S a$ and pseudospectral acceleration Spa is significantly affected by variations in the seismological parameters. The spectral absolute acceleration $S a$ approaches the pseudospectral acceleration Spa with increasing moment magnitudes and source-to-site distances, particularly for longer oscillator periods.

3. The effects of site conditions on the relationships between the two spectra are dependent on specific site parameters, and the fundamental period of the site has a significant effect on the Sa/Spa ratio.

4. The key factor governing the seismological effects is the frequency content of the ground motions. The observed phenomena can be reasonably explained by the variation of the frequency content with the seismological parameters.

5. The proposed simple $S a / S p a$ formulation is demonstrated to perform very well for a wide range of periods utilized in practical seismic design by comparing with the results of real seismic records.

\section{Acknowledgments}

This study was partially supported by the National Natural Science Foundation of China (Grant No. 51738001); the support is gratefully acknowledged. The authors are also grateful to Baojian Hang for help with illustration of the figures in this paper.

\section{Declarations}


422 Funding The research leading to these results received funding from the National Natural

423 Science Foundation of China (Grant No. 51738001).

424 Conflicts of interest/Competing interests The authors have no conflicts of interest to 425 declare that are relevant to the content of this article.

426 Data availability All data generated or analyzed during this study are included in this 427 published article.

428 Code availability Available upon request.

429 Authors' contributions Haizhong Zhang: conceptualization, methodology, writing-original 430 draft preparation, investigation. Yan-Gang Zhao: data curation, visualization, supervision, 431 writing-reviewing and editing.

\section{References}

434 Boore, D.M., 1983. Stochastic simulation of high-frequency ground motions based on seismological models of the radiated spectra. Bulletin of the Seismological Society of America 736, 1865-1894.

Boore, D.M., 2003. Simulation of ground motion using the stochastic method. Pure and Applied Geophysics 60, 635-676.

Boore, D.M., 2005. SMSIM-Fortran programs for simulating ground motions from earthquakes: version 2.3, U.S. Geol. Surv. Open-File Rept. OFR 96-80-A, Menlo Park, California.

Boore, D.M. and Thompson, E.M., 2014. Path durations for use in the stochastic-method simulation of ground motions. Bulletin of the Seismological Society of America 104,

Boore, D.M. and Thompson, E.M., 2015. Revisions to some parameters used in stochastic-method simulations of ground motion. Bulletin of the Seismological Society of 
America 105, 1029-1041.

448 Cartwright, D.E. and Longuet-Hlggms, M.S., 1956. The statistical distribution of the maxima of a random function. Proceedings of the Royal Society of London. Series A, Mathematical and Physical Sciences 237, 212-223.

Chopra, A.K., 2007. Elastic response spectrum: A historical note. Earthquake Engineering and Structural Dynamics 36, 3-12.

Davenport, A.G., 1964. Note on the distribution of the largest value of a random function with application to gust loading. Proceedings of the Institution of Civil Engineers 28, 187-196.

Jenschke, V.A., Clough, R.W., and Penzien, J., 1964. Analysis of earth motion accelerograms. Report No. SESM 64-1, University of California, Berkeley, CA.

Jenschke, V.A., Clough, R.W., and Penzien, J., 1965. Characteristics of strong ground motions. Proceedings of the Third World Conference on Earthquake Engineering III, New Zealand, $125-142$.

Lin, Y.Y. and Chang, K.C., 2003. A study on damping reduction factor for buildings under earthquake ground motion. Journal of Structural Engineering 129(2), 206-214.

Mentrasti, L., 2008. Estimate of spectral and pseudo-spectral acceleration proximity. Engineering Structures 30(9), 2338-2346.

Nigam, N. and Jennings, P., 1969. Calculation of response spectra from strong-motion earthquake records. Bulletin of the Seismological Society of America 59(2), 909-922.

Newmark, N.M. and Rosenblueth, E., 1972. Fundamentals of earthquake engineering. Englewood Cliffs: Prentice Hall.

Papagiannopoulos, G.A., Hatzigeorgiou, G.D., and Beskos, D.E, 2013. Recovery of spectral absolute acceleration and spectral relative velocity from their pseudo-spectral counterparts. Earthquakes and Structures 4 (5), 1-000.

Pu, W., Kasai, K., Karoki, E.K., and Huang, B., 2016. Evaluation of the damping modification 
factor for structures subjected to near-fault ground motions. Bulletin of Earthquake Engineering 14, 1519-1544.

Sadek, F., Mohraj, B., and Riley, M.A., 2000. Linear static and dynamic procedures for structures with velocity-dependent dampers. Journal of Structural Engineering 126(8), $887-895$.

Song, J., Chu, Y.L., Liang, Z., and Lee, G.C., 2007. Estimation of peak relative velocity and peak absolute acceleration of linear SDOF systems. Earthquake Engineering \& Engineering Vibration 6(1), 1-10.

Vanmarcke, E.H., 1975. On the distribution of the first-passage time for normal stationary random processes. Journal of Applied Mechanics 42, 215-220.

Veletsos, A.S. and Newmark, N.M., 1964. Response spectra for single-degree-of freedom elastic and inelastic systems. Report No. RTD-TDR-63-3096, vol. III, Air Force Weapons Laboratory, Albuquerque, NM.

Wang, X. and Rathje, E.M., 2016. Influence of peak factors on site amplification from random vibration theory based site-response analysis. Bulletin of the Seismological Society of America 106, 1-14.

Zhang, D., Bai, Y., and Gao, J., 2016. Probe into several important concepts in Chinese current seismic response spectra. Journal of Building Structures 37, 1000-6869. (In Chinese)

Zhang, H.Z., Saito, T., and Zhao, Y.G., 2017. Simple calculation method of seismic motion amplification ratio corresponding to fundamental period. Journal Structural and Construction Engineering AIJ 82, 597-604. (In Japanese)

Zhang, H.Z. and Zhao, Y.G., 2018. A simple approach for estimating the first resonance peak of layered soil profiles. Journal of Earthquake and Tsunami 12, 185005.

Zhang, H.Z. and Zhao, Y.G., 2020. Damping modification factor based on random vibration theory using a source-based ground-motion model. Soil Dynamics and Earthquake 
499

500

501

502

503

504 km, (c) $M=5, R=126.20 \mathrm{~km}$, and (d) $M=7, R=126.20 \mathrm{~km}$.

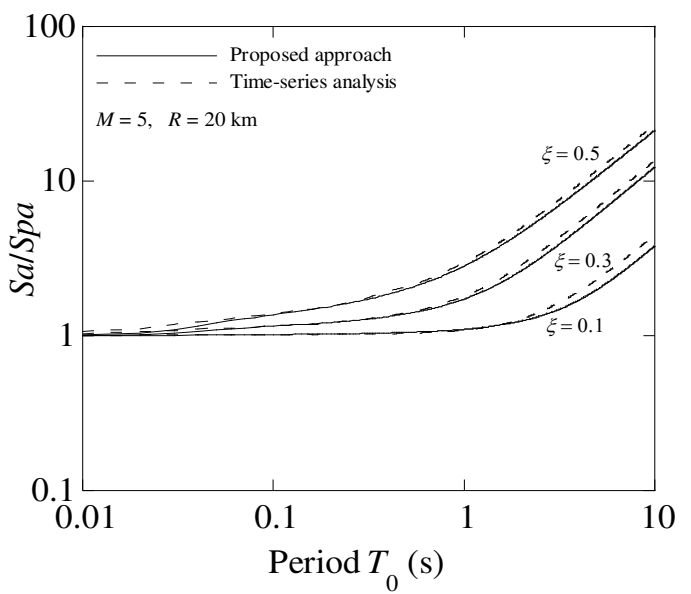

(a)

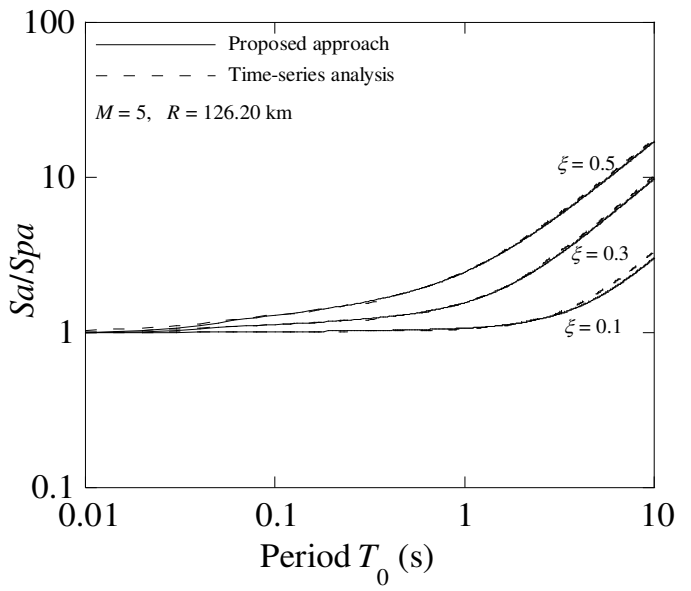

(c)

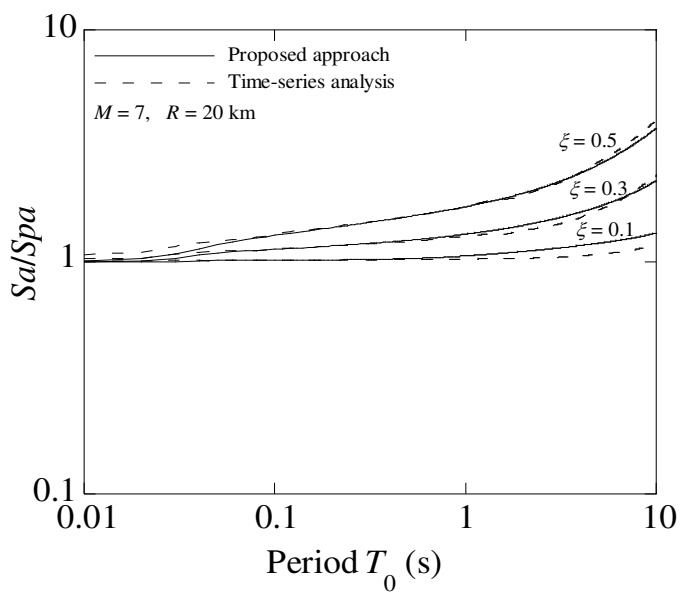

(b)

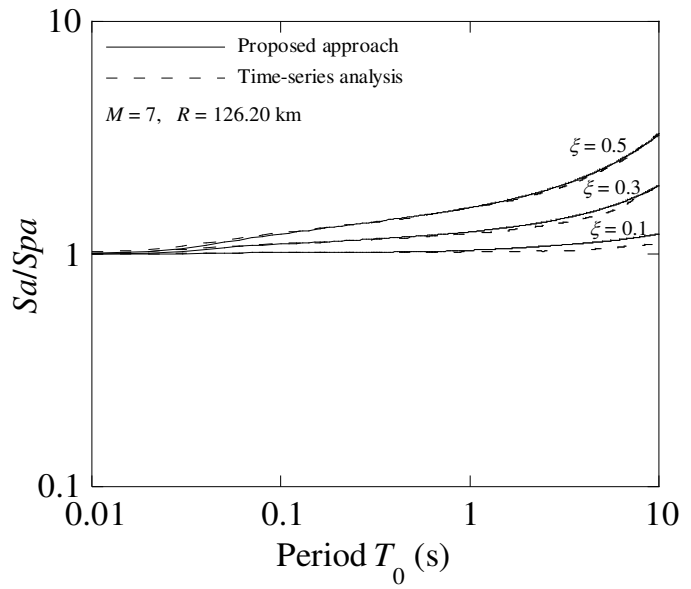

(d)

Figure 1. Variation of spectral ratio Sa/Spa with oscillator damping ratio $\xi$ for cases with moment magnitude $M$ and site-to-source distance $R$ of (a) $M=5, R=20 \mathrm{~km}$, (b) $M=7, R=20$ 


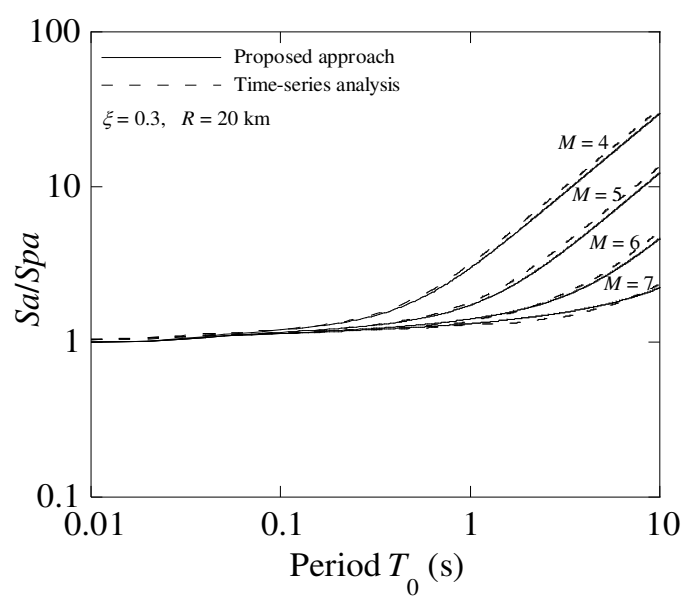

(a)

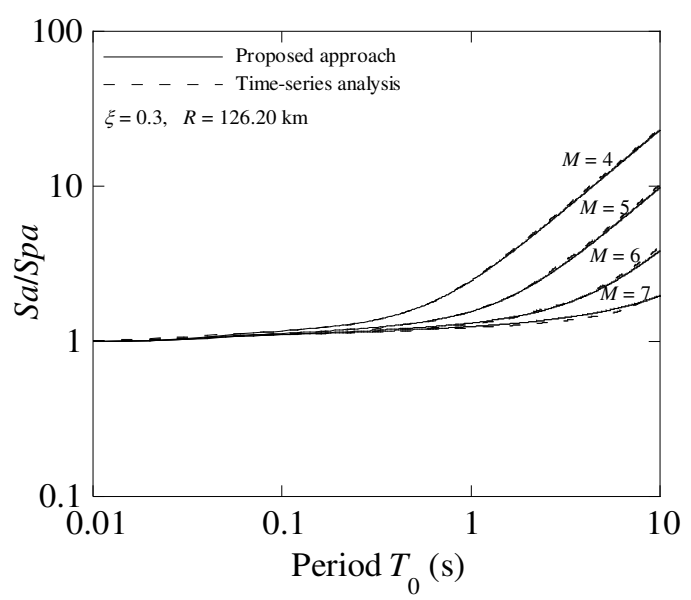

(c)

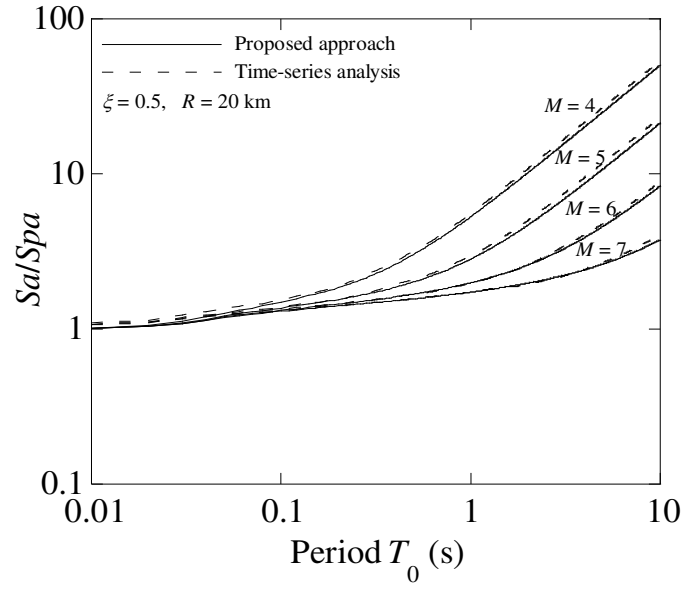

(b)

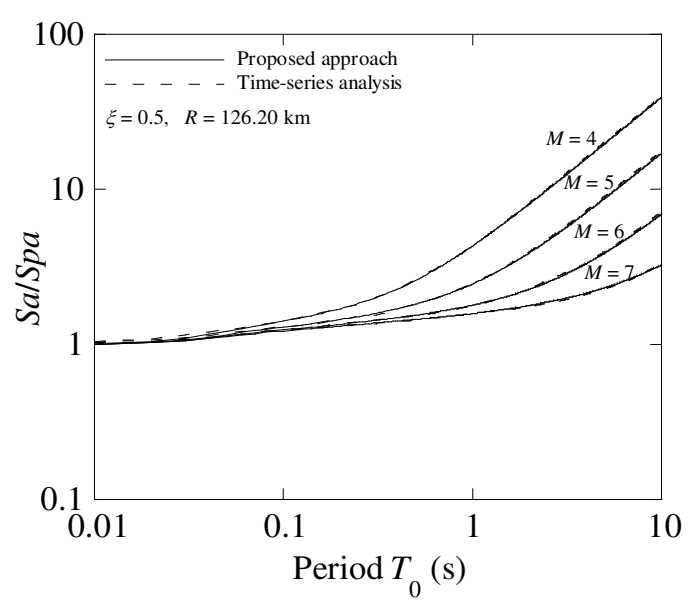

(d)

511 Figure 2. Variation of spectral ratio $S a / S p a$ with moment magnitude $M$ for cases with oscillator

512 damping ratio $\xi$ and site-to-source distance $R$ of (a) $\xi=0.3, R=20 \mathrm{~km}$, (b) $\xi=0.5, R=20 \mathrm{~km}$,

513

(c) $\xi=0.3, R=126.20 \mathrm{~km}$, and (d) $\xi=0.5, R=126.20 \mathrm{~km}$.

514 


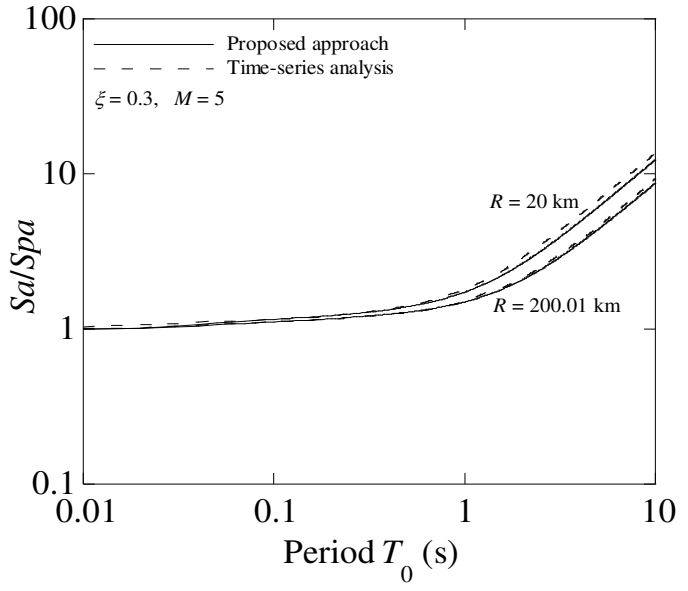

(a)

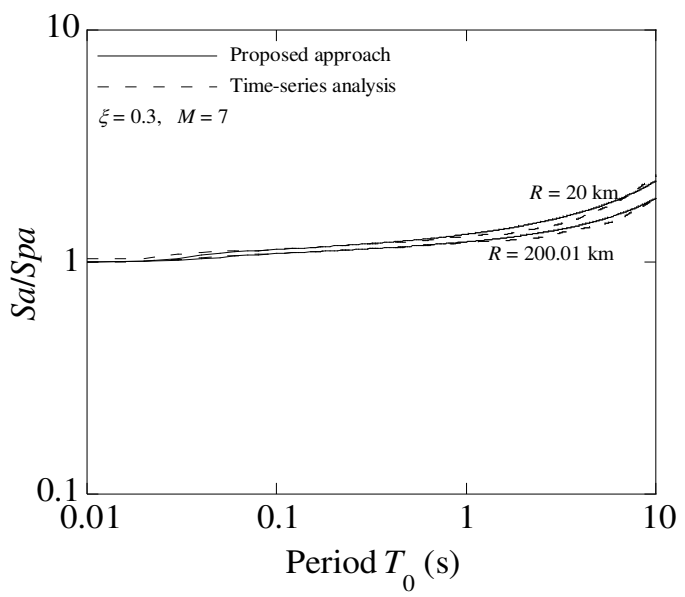

(c)

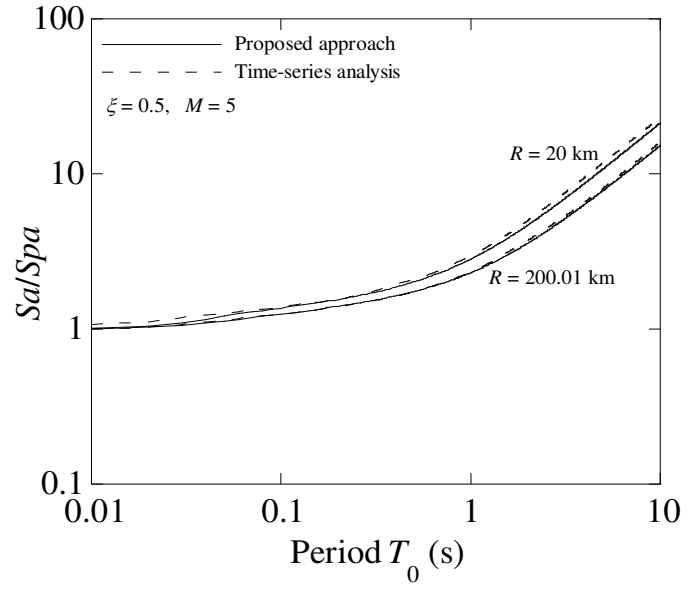

(b)

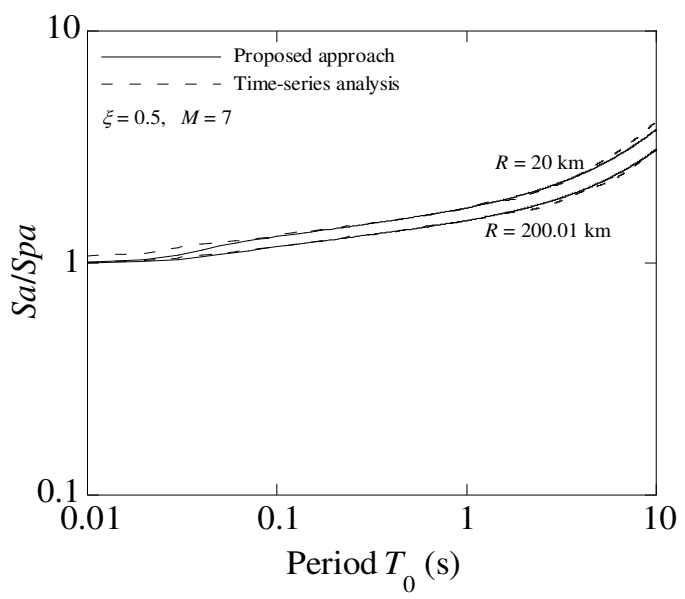

(d)

Figure 3. Variation of spectral ratio $S a / S p a$ with site-to-source distance $R$ for cases with 


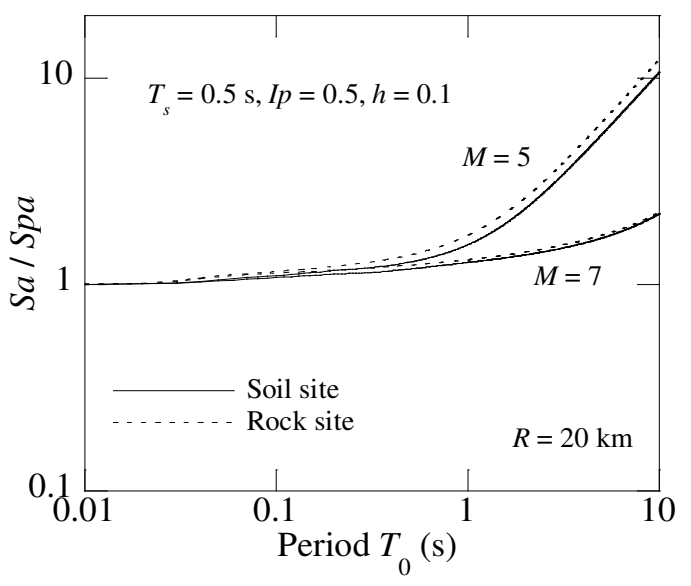

(a)

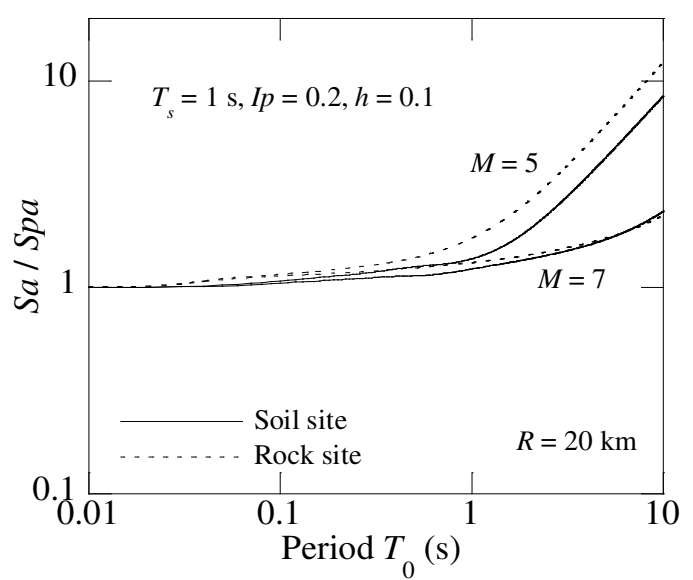

(c)

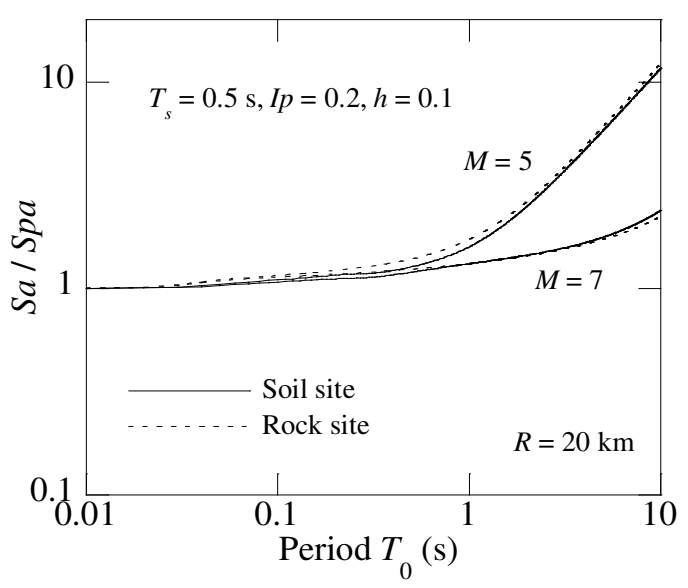

(b)

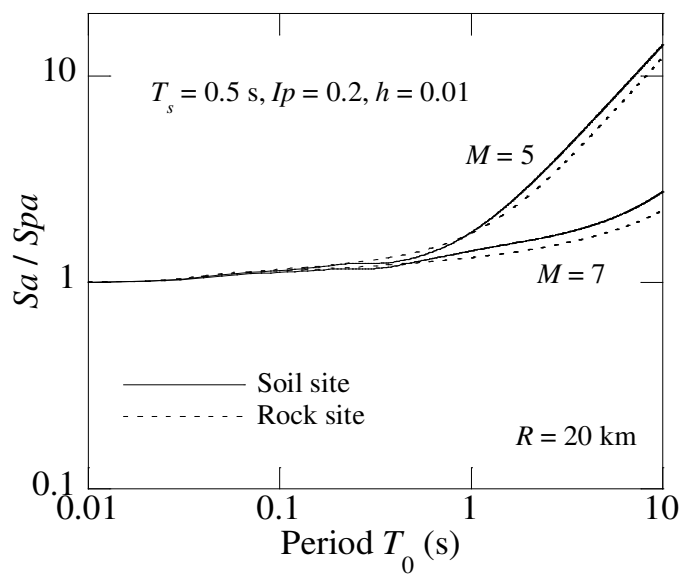

(d)

Figure 4. Variation of spectral ratio $S a / S p a$ with site parameters for soil sites with fundamental period $T_{s}$ impedance ratio $I p$, and soil damping ratio $h$ of (a) $T_{s}=0.5 \mathrm{~s}, I p=0.5, h=0.1$, (b) $T_{s}$
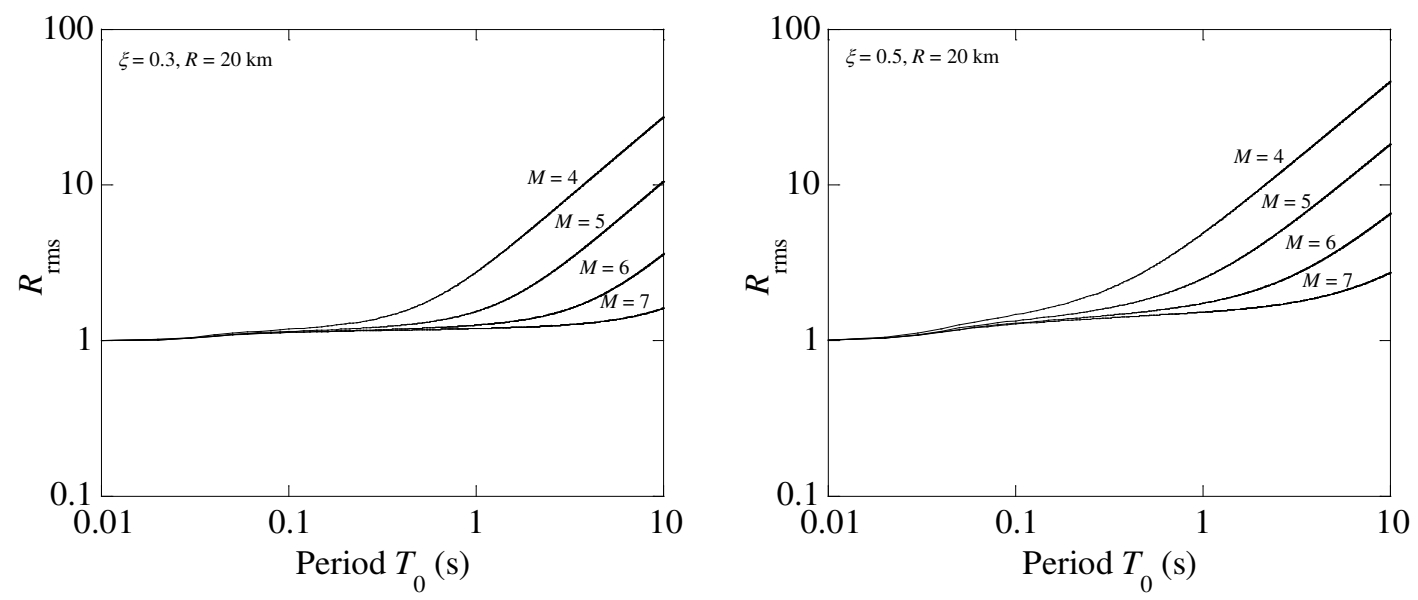
(a)

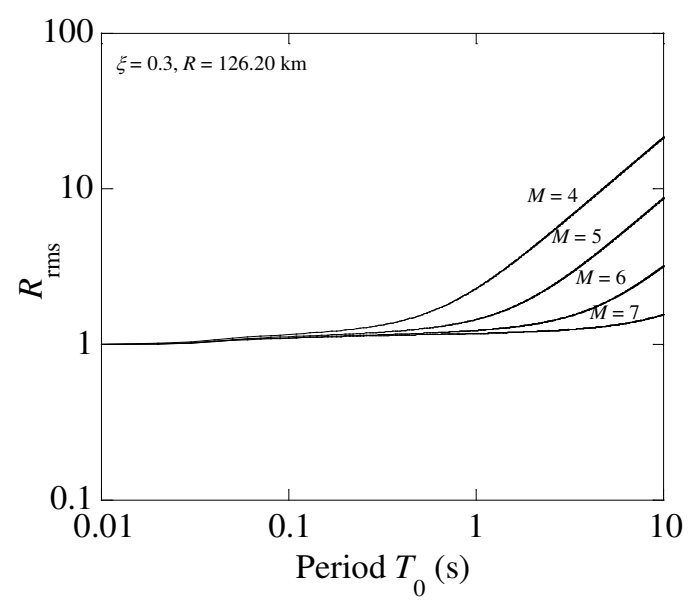

535

536

537

538

539

540

541

542

(c)

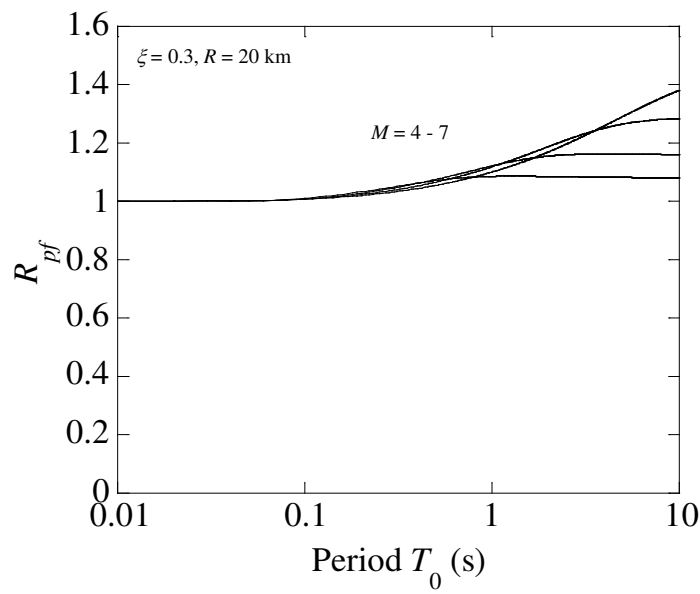

(a) (b)

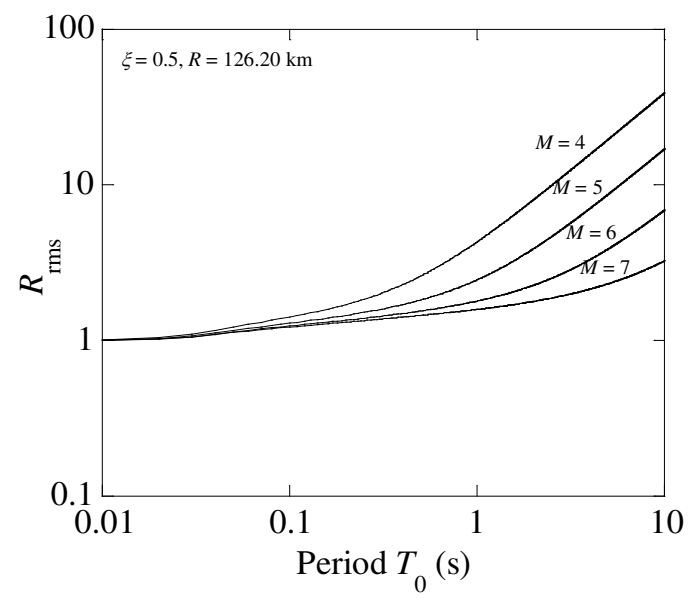

(d)

Figure 5. Variation of the first term with moment magnitude $M$ for cases with oscillator damping ratio $\xi$ and site-to-source distance $R$ of (a) $\xi=0.3, R=20 \mathrm{~km}$, (b) $\xi=0.5, R=20 \mathrm{~km}$, (c) $\xi=0.3, R=126.20 \mathrm{~km}$, and (d) $\xi=0.5, R=126.20 \mathrm{~km}$.

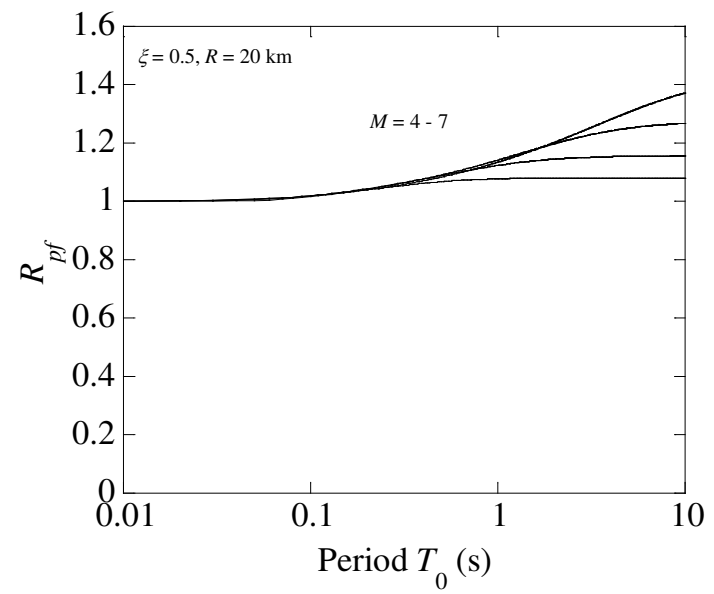

(b) 


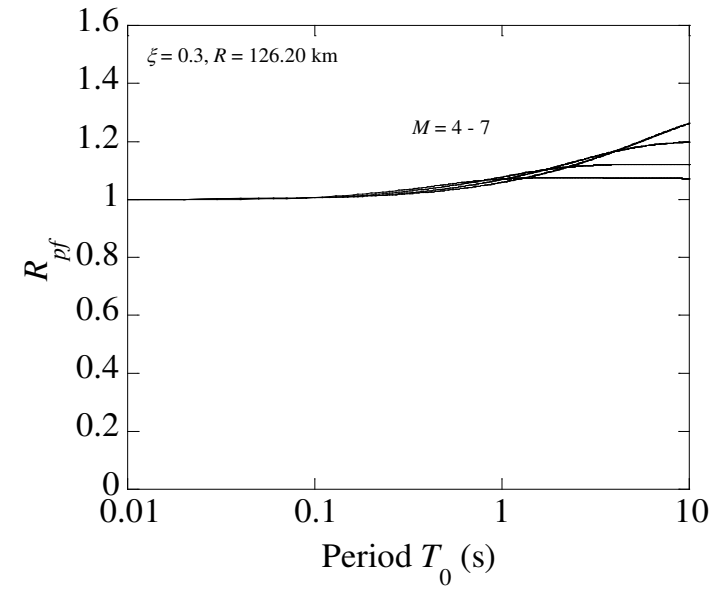

(c)

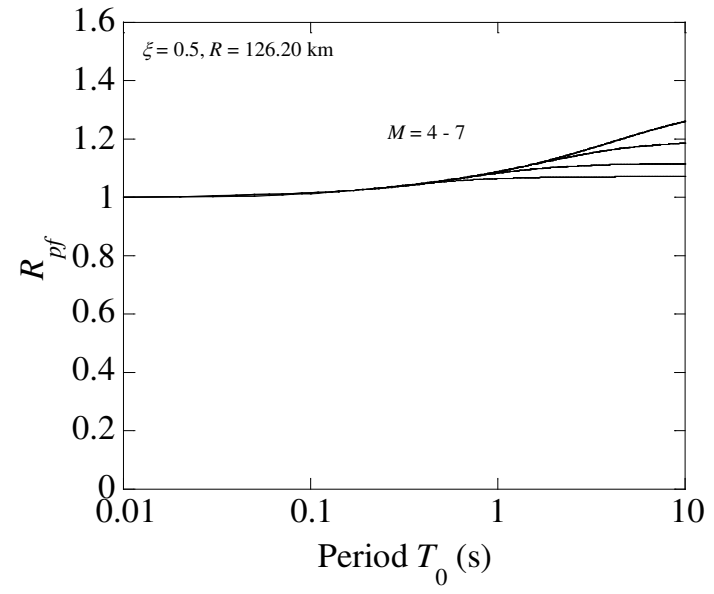

(d)

545 Figure 6. Variation of the second term with moment magnitude $M$ for cases with oscillator

546 damping ratio $\xi$ and site-to-source distance $R$ of (a) $\xi=0.3, R=20 \mathrm{~km}$, (b) $\xi=0.5, R=20 \mathrm{~km}$,

$547 \quad$ (c) $\xi=0.3, R=126.20 \mathrm{~km}$, and (d) $\xi=0.5, R=126.20 \mathrm{~km}$.

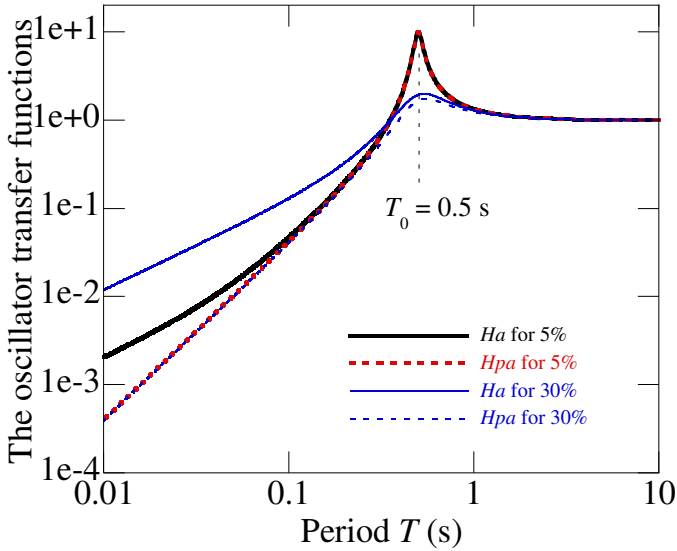

Figure 7. Comparison of oscillator transfer functions for spectral and pseudospectral accelerations for two oscillator damping ratios (5\% and 30\%).

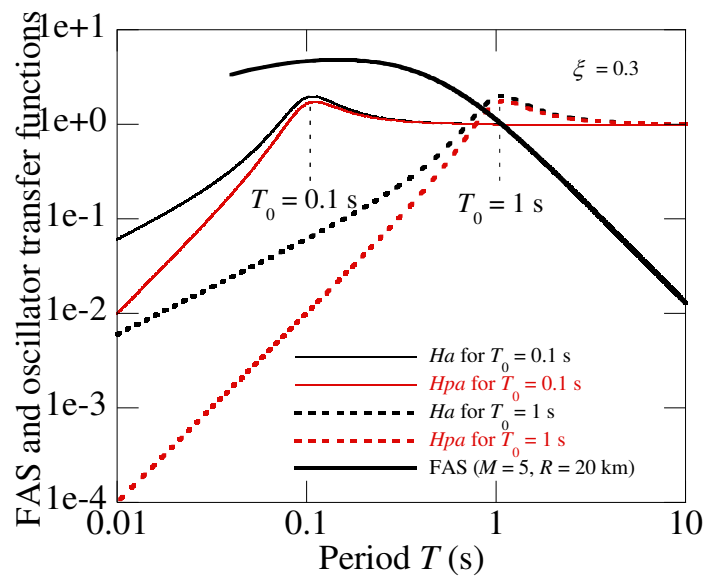


552 Figure 8. Comparison of oscillator transfer functions for spectral and pseudospectral accelerations for different oscillator periods $(0.1 \mathrm{~s}$ and $1 \mathrm{~s})$.

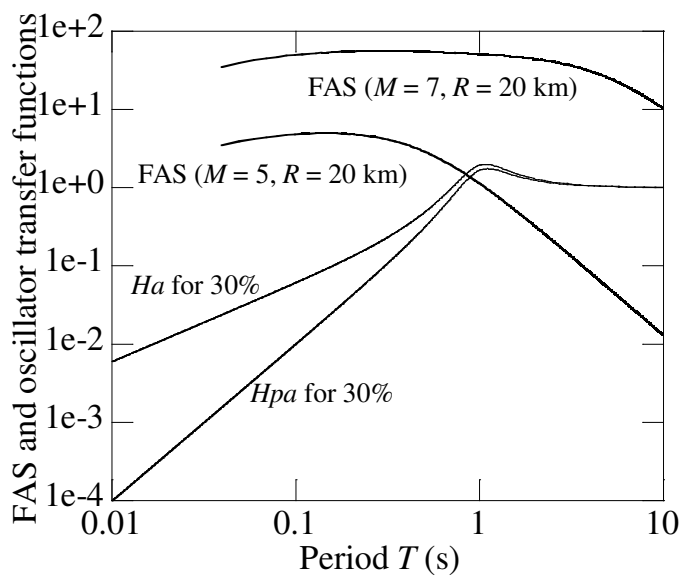

(a)

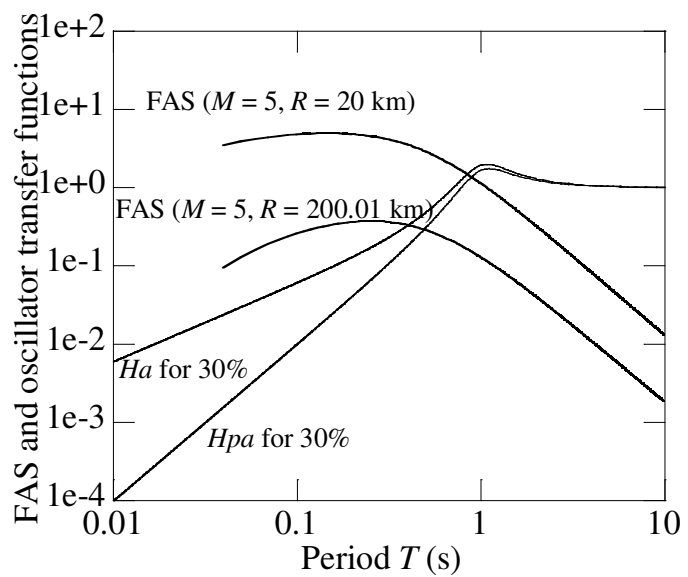

(b)

Figure 9. Variation of the frequency content of ground-motion FAS with (a) moment magnitude and (b) site-to-source distance.

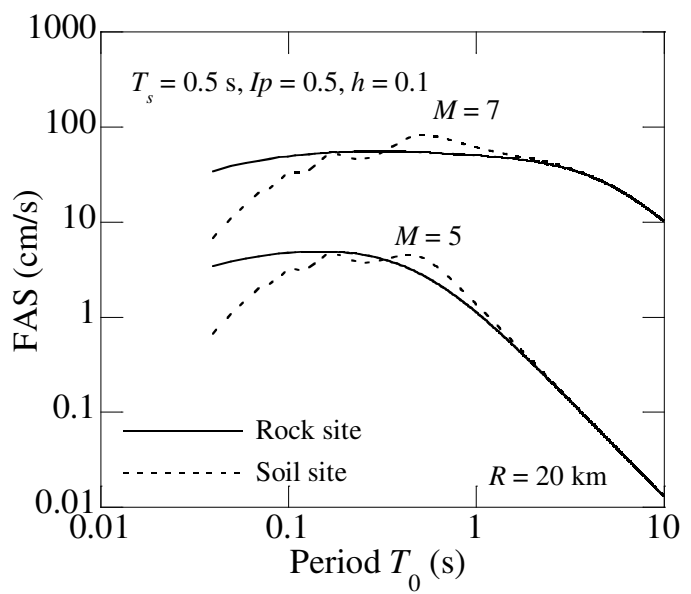

(a)

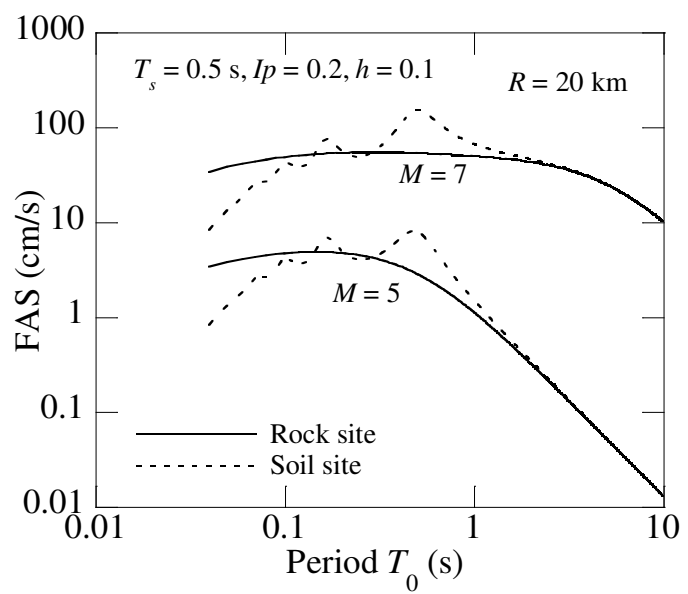

(b) 
561

562

563

564

565

566

567

568

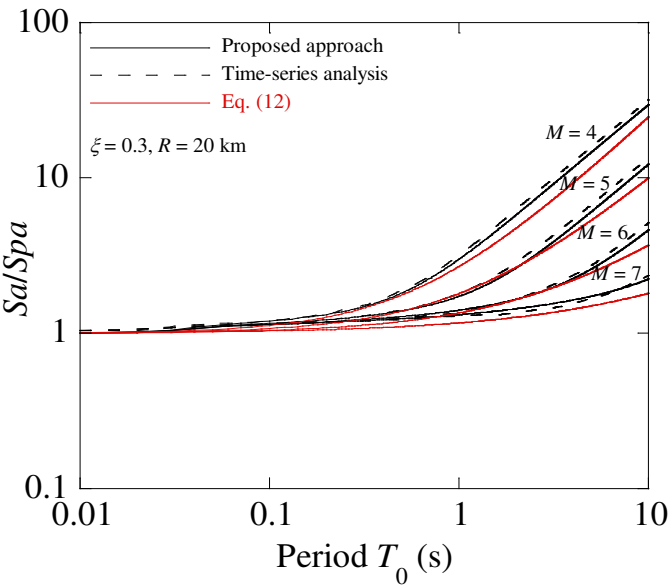

(a)

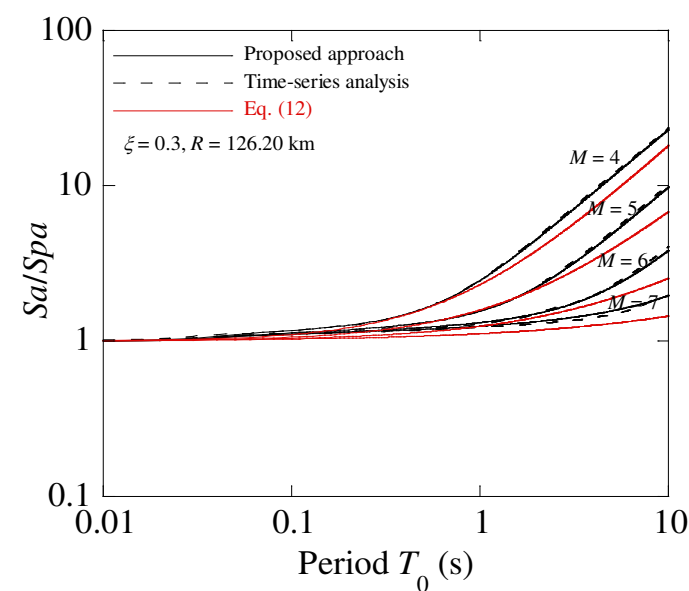
(d) $T s=0.5 \mathrm{~s}, I p=0.2, h=0.01$.

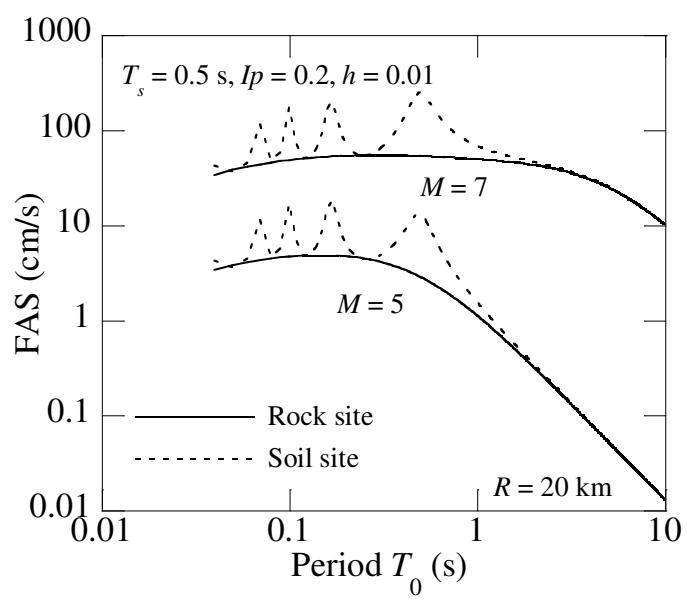

(d)

Figure 10. Variations of the frequency content of ground-motion FAS with site conditions for (a) $T s=0.5 \mathrm{~s}, I p=0.5, h=0.1$, (b) $T s=0.5 \mathrm{~s}, I p=0.2, h=0.1$, (c) $T s=1 \mathrm{~s}, I p=0.2, h=0.1$, and

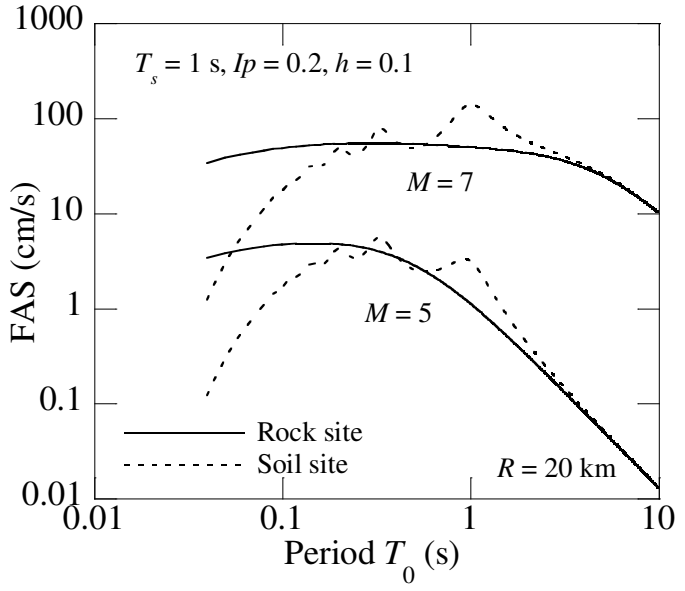

(c) 
571 Figure 11. Comparisons of $S a / S p a$ results from the proposed approach, time-series analysis, 572 and Eq. (12) for the cases with oscillator damping ratio $\xi$ and site-to-distance $R$ of (a) $\xi=0.3, R$ $573=20 \mathrm{~km}$, (b) $\xi=0.5, R=20 \mathrm{~km}$, (c) $\xi=0.3, R=126.20 \mathrm{~km}$, and (d) $\xi=0.5, R=126.20 \mathrm{~km}$.

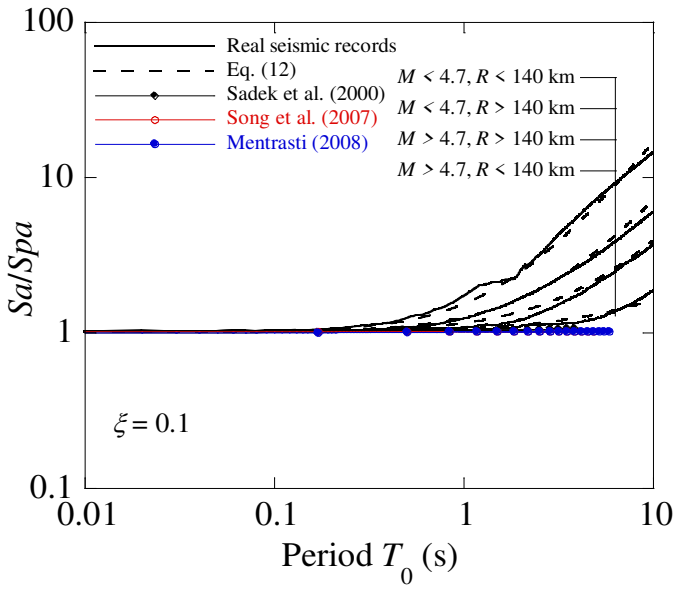

(a)

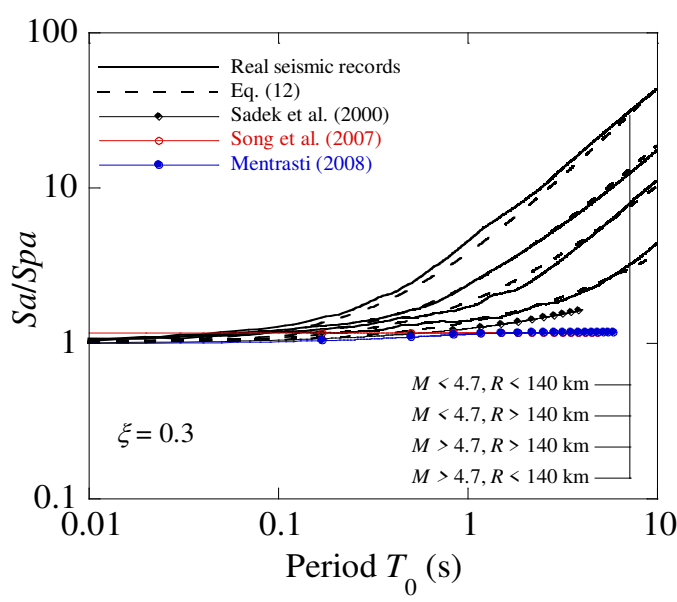

(c)

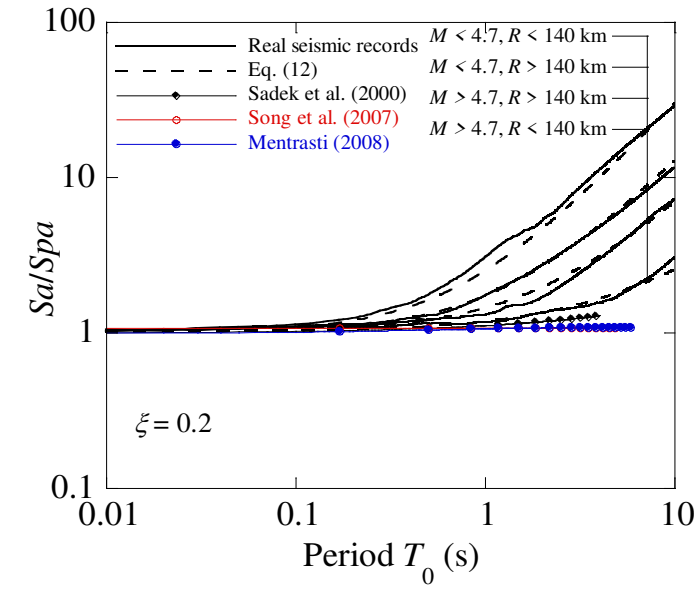

(b)

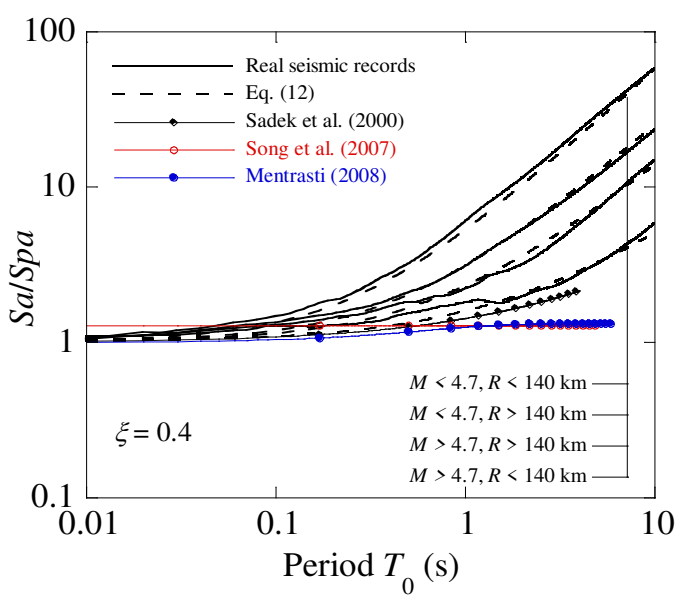

(d)

580 Figure 12. Comparisons of Sa/Spa results from Eq. (12), real seismic records, and the 581 formulations of Sadek et al. (2000), Song et al. (2007), and Mentrasti (2008) for the cases with oscillator damping ratio $\xi$ of (a) $\xi=0.1$, (b) $\xi=0.2$, (c) $\xi=0.3$, and (d) $\xi=0.4$. 